\title{
Call for papers - 2020 special issue
}

\section{Problematic substance use in Canada: trends and emerging issues in public health}

Editors: Robert Geneau and Margaret de Groh (Editors-in-Chief, Public Health Agency of Canada)

Substances, such as alcohol, cannabis and other drugs pose serious challenges for public health, public safety and the health and well-being of Canadians through their potential to cause dependence, illness and/or harm.

With Canada continuing to face a national opioid crisis, changes to federal legislation on cannabis, significant societal costs associated with alcohol and tobacco, and growing popularity of vaping products, it is imperative that we monitor the scope and impacts of problematic substance use through a public health lens. The goal of this special issue is to provide the latest research evidence to inform the Canadian Drugs and Substances Strategy and to share timely scientific findings with decision-makers, service providers, communities and those living with or affected by problematic substance use.

Health Promotion and Chronic Disease Prevention in Canada: Research, Policy and Practice is seeking relevant topical research articles that:

- $\quad$ Characterize the current state of problematic substance use, polyuse and substance use disorders in Canada;

- $\quad$ Examine trends or explore emerging issues in problematic use of cannabis, opioids, alcohol, tobacco and other emerging substances;

- $\quad$ Synthesize and/or review evidence on substance-related policies and interventions in the Canadian context.

Article submissions may also address at-risk, marginalized or under-studied populations, stigma and discrimination, social determinants, lifecourse epidemiology, marketing, retail and accessibility of substances, and prevention across different settings. Substances of interest include cannabis, alcohol, tobacco, opioids, and other psychoactive substances. In addition, we welcome articles that examine the use of new e-cigarette ("vaping") products.

Refer to our website for information on invited article types and detailed submission guidelines for authors: https://www.canada.ca Len/public-health/services/reports-publications/health-promotion-chronic-disease-prevention-canada-research-policy-practice ¿information-authors.html. For any pre-submission questions about suitability or scope, please direct inquiries to $\underline{\text { PHAC.HPCDP.Journal }}$ -Revue.PSPMC.ASPC@canada.ca.

Submission Deadline: Kindly refer to this call for papers in your submission covering letter and submit manuscripts by email to PHAC.HPCDP.Journal-Revue.PSPMC.ASPC@canada.ca by September 3 ${ }^{\text {rd }}, 2019$. 